\title{
FORMAS NORMALES DE ECUACIONES DIFERENCIALES FORMALES
}

\author{
Renato Benazic Tomé ${ }^{1}$ \\ Soledad Ramírez Carrasco ${ }^{2}$
}

RESUMEN.- En este artículo se transforma un sistema de ecuaciones diferenciales ordinarias asociado a un campo formal, en otro sistema más simple, correspondiente a su "forma canónica" mediante un cambio de coordenadas formal. En particular se demuestra la versión formal del Teorema de Linealización de Poincaré y del Teorema de Poincaré-Dulac.

PALABRAS CLAVE: Formas normales, Ecuaciones diferenciales formales, dinámica compleja.

\section{NORMAL FORMS OF THE FORMAL DIFFĖRENTIAL EQUATIONS}

\begin{abstract}
In this paper, we resolve the problem of finding a formal change of coordinates between formal vector field and another more simple (its "canonical form»). In particular, we proof the Poincarés theorem of formal linearization and the Poincaré-Dulac theorem.
\end{abstract}

KEYWORDS: Normal forms, Formal differential equations, Complex Dynamics.

\section{PRELIMINARES}

El objetivo del presente trabajo es determinar algunas formas normales formales de Ecuaciones Diferenciales. Entendemos por forma normal a una expresión más simple de un Sistema de Ecuaciones Diferenciales dado, obtenido por un cambio de coordenadas y que conserve las principales propiedades cualitativas de las curvas solución del sistema dado. A manera de ejemplo, consideremos el Sistema,

$$
\mid \begin{aligned}
& z_{1}^{\prime}=\lambda_{1} z_{1}+a_{1,2,0} z_{1}^{2}+a_{1,1,1} z_{1} z_{2}+a_{1,0,2} z_{2}^{2}+\ldots \\
& z_{2}^{\prime}=\lambda_{2} z_{2}+a_{2,2,0} z_{1}^{2}+a_{2,1,1} z_{1} z_{2}+a_{2,0,2} z_{2}^{2}+\ldots
\end{aligned}
$$

donde $\lambda_{1}, \lambda_{2} \in \mathbb{C}^{*}=\mathbb{C}-\{0\}$ y $a_{j, 2,0} z_{1}^{2}+a_{j, 1,1} z_{1} z_{2}+a_{j, 0,2} z_{2}^{2}+\ldots(j=1,2)$ son series de dos variables complejas, convergentes en una vecindad del origen de $\mathbb{C}^{2}$ y $z^{\prime}=\frac{d z}{d T}$ con $T \in \mathbb{C}$.

'Universidad Nacional Mayor de San Marcos. Facultad de Ciencias Matemáticas. Instituto de Matemática y Ciencias Afines-IMCA. mail: benazic@uni.edu.pe

${ }^{2}$ Universidad Nacional Mayor de San Marcos. Facultad de ciencias Matemáticas. 
Vamos a suponer que el segmento de recta que une los números complejos $\lambda_{1}$ y $\lambda_{2}$ no contienen al origen.

Si $\frac{\lambda_{1}}{\lambda_{2}}, \frac{\lambda_{2}}{\lambda_{1}} \notin\{2,3, \ldots\}$, entonces en Benazic [2] se prueba que existe un cambio de coordenadas holomorfo

$$
\mid \begin{aligned}
& z_{1}=w_{1}+b_{1,2,0} w_{1}^{2}+b_{1,1,1} w_{1} w_{2}+b_{1,0,2} w_{2}^{2}+\ldots \\
& z_{2}=w_{2}+b_{2,2,0} w_{1}^{2}+b_{2,1,1} w_{1} w_{2}+b_{2,0,2} w_{2}^{2}+\ldots
\end{aligned}
$$

que transforma (1) en el Sistema Lineal

$$
\mid \begin{aligned}
& w_{1}^{\prime}=\lambda_{1} w_{1} \\
& w_{2}^{\prime}=\lambda_{2} w_{2}
\end{aligned} .
$$

En este caso, decimos que (3) es la forma normal holomorfa del Sistema (1).

En cambio, si $\lambda_{1}=n \lambda_{2}$ con $n \geq 2$, entonces (ver Benazic [2]) existe un cambio de coordenadas convergente del tipo (2) que transforma el sistema (1) en

$$
\mid \begin{aligned}
& w_{1}^{\prime}=\lambda_{1} w_{1}+c w_{2}^{n} \\
& w_{2}^{\prime}=\lambda_{2} w_{2}
\end{aligned}
$$

Así, bajo estas condiciones sobre $\lambda_{1}$ y $\lambda_{2}$, se tiene que (4) es la forma normal holomorfa de (1).

Existen formas normales holomorfas para sistemas de $n$ ecuaciones diferenciales que presentan términos de orden 1 (ver Camacho [4], [5], [6]). Cuando no existen términos de orden 1, es posible, bajo ciertas condiciones (ver Benazic [3]), obtener formas canónicas holomorfas.

Siguiendo las ideas de Arnold [1], vamos a obtener formas canónicas formales (es decir el cambio de coordenadas está dado por una serie formal de potencias) de un sistema formal de $n$ ecuaciones diferenciales ordinarias. En la Sección 2, definimos y estudiamos las principales propiedades de las series formales. En la Sección 3, estudiamos los Campos Vectoriales Formales y establecemos algunos resultados útiles sobre cambio de variables. En la Sección 4, asociamos a cada Campo Vectorial Formal un Sistema de ecuaciones Diferenciales Ordinarias y se define el importante concepto de resonancia. Finalmente, en la sección 5 enunciamos y demostramos los teoremas que garantizan la existencia de formas normales formales.

\section{SERIES DE POTENCIAS FORMALES}

En este artículo, utilizamos la notación de los multi-índices de Schwartz. Un multi-índice de dimensión $n$ es una $n$ - upla $\alpha=\left(\alpha_{1}, \ldots, \alpha_{n}\right)$ de enteros no negativos. La norma de $\alpha$ se denota con $|\alpha|$ y se define como $|\alpha|=\alpha_{1}+\ldots+\alpha_{n}$. Observe que, $|\alpha| \geq 0$ para todo multi-índice $\alpha$.

Si $z=\left(z_{1}, \ldots, z_{n}\right)$ es una $n$ - upla (de números complejos o más generalmente de elementos de un anillo) y $\alpha=\left(\alpha_{1}, \ldots, \alpha_{n}\right)$ es un multi-índice, definimos, 


$$
z^{\alpha}=z_{1}^{\alpha_{1}} \ldots z_{n}^{\alpha_{n}}
$$

Denotaremos por, $\mathbb{C}\left[\left[z_{1}, \ldots, z_{n}\right]\right]$ al conjunto de todas las series de potencias formales con coeficientes en $\mathbb{C}$, es decir, $S \in \mathbb{C}\left[\left[z_{1}, \ldots, z_{n}\right]\right]$ si y sólo si $S$ es de la forma

$$
S=\sum_{|\alpha| \geq 0}^{\infty} a_{\alpha} z^{\alpha}
$$

donde, $a_{\alpha} \in \mathbb{C}$ para todo $\alpha$ multi-índice. Observe que toda serie de potencias formal puede ser escrita como una suma de polinomios homogéneos. En efecto, si definimos

$$
P_{k}(z)=\sum_{|\alpha|=k} a_{\alpha} z^{\alpha}
$$

se sigue inmediatamente que, $P_{k}$ es un polinomio homogéneo en las variables $z_{1}, \ldots, z_{n}$ de grado $k \mathrm{y}$

$$
S=\sum_{k \geq 0} P_{k}(z)
$$

Desde este punto de vista, una serie formal es una generalización de un polinomio. Más específicamente, denotemos por $\mathcal{P}=\mathbb{C}\left[z_{1}, \ldots, z_{n}\right]$ (respectivamente, $\mathcal{H}_{r}=\mathcal{H}_{r}\left[z_{1}, \ldots, z_{n}\right]$, con $r=0,1,2, \ldots$ ) al conjunto de todos los polinomios (respectivamente, polinomios homogéneos de grado $r$ ) en las variables $z_{1}, \ldots, z_{n}$ y con coeficientes en $\mathbb{C}$, es decir,

$$
\begin{gathered}
\mathcal{H}_{r}=\left\{\sum_{|\alpha|=r} a_{\alpha} z^{\alpha}: a_{\alpha} \in \mathbb{C},\right\} \\
\mathcal{P}=\left\{\sum_{j=0}^{n} P_{j}: P_{j} \in \mathcal{H}_{j}, \forall 0 \leq j \leq n\right\} .
\end{gathered}
$$

Claramente,

$$
\mathcal{H}_{r} \subseteq \mathbb{C}\left[\left[z_{1}, \ldots, z_{n}\right]\right] \text { y } \mathcal{P} \subseteq \mathbb{C}\left[\left[z_{1}, \ldots, z_{n}\right]\right]
$$

Sean $S, T \in \mathbb{C}\left[\left[z_{1}, \ldots, z_{n}\right]\right]$, si $S=\sum_{|\alpha| \geq 0}^{\infty} a_{\alpha} z^{\alpha}, T=\sum_{|\alpha| \geq 0}^{\infty} b_{\alpha} z^{\alpha}$, entonces la suma formal de $S \mathrm{y}$ $T$ denotada como $S+T$ se define como,

$$
S+T=\sum_{|\alpha| \geq 0}^{\infty}\left(a_{\alpha}+b_{\alpha}\right) z^{\alpha}
$$

Si $c \in \mathbb{C}$ entonces el producto de la serie formal $S$ por el complejo $c$, denotado por $c S$, se define como 


$$
c S=\sum_{|\alpha| \geq 0}^{\infty}\left(c a_{\alpha}\right) z^{\alpha} .
$$

Con estas dos operaciones $\mathbb{C}\left[\left[z_{1}, \ldots, z_{n}\right]\right]$ se torna un espacio vectorial sobre $\mathbb{C}$. Más aún, $\mathcal{H}_{r}$ y $\mathcal{P}$ son $\mathbb{C}$-subespacios vectoriales de $\mathbb{C}\left[\left[z_{1}, \ldots, z_{n}\right]\right]$ además

$$
\mathbb{C}\left[\left[z_{1}, \ldots, z_{n}\right]\right]=\underset{r \geq 0}{\bigoplus} \mathcal{H}_{r}
$$

Sean $S=\sum_{k \geq 0} P_{k}, T=\sum_{k \geq 0} Q_{k} \in \mathbb{C}\left[\left[z_{1}, \ldots, z_{n}\right]\right]=\underset{k \geq 0}{\oplus} \mathcal{H}_{k}$, el producto de $S$ y $T$ se define como

$$
S \cdot T=\sum_{n \geq 0}\left(\sum_{k=0}^{n} P_{n-k} Q_{k}\right) .
$$

No es difícil probar que, con las operaciones de suma y producto de series, $\mathbb{C}\left[\left[z_{1}, \ldots, z_{n}\right]\right]$ es un anillo.

Sea $S=\sum_{k \geq 0} P_{k} \in \mathbb{C}\left[\left[z_{1}, \ldots, z_{n}\right]\right]$ el orden de $S$, denotado por $\operatorname{ord}(S)$ es por definición el menor número entero no negativo $r$ tal que $P_{r} \in \mathcal{H}_{r}$ no es idénticamente cero, es decir

$$
\operatorname{ord}(S)=r \Leftrightarrow P_{0} \equiv \cdots \equiv P_{r-1} \equiv 0 \text { y } P_{r} \not 00 .
$$

Es claro que, se cumplen las siguientes propiedades:

1. $\operatorname{ord}(S+T) \geq \min \{\operatorname{ord}(S), \operatorname{ord}(T)\}, \forall S, T \in \mathbb{C}\left[\left[z_{1}, \ldots, z_{n}\right]\right]$ no nulos.

2. $\operatorname{ord}(c S)=\operatorname{ord}(S), \forall S \in \mathbb{C}\left[\left[z_{1}, \ldots, z_{n}\right]\right]$ no nulo y $\forall c \in \mathbb{C}$ no nulo.

3. $\operatorname{ord}(S \cdot T)=\operatorname{ord}(S)+\operatorname{ord}(T), \forall S, T \in \mathbb{C}\left[\left[z_{1}, \ldots, z_{n}\right]\right]$ no nulos.

La derivada parcial con respecto a la variable $z_{j}$ de la serie formal $S=\sum_{k \geq 0} P_{k}(z)$ se define como,

$$
\frac{\partial S}{\partial z_{j}}=\sum_{k \geq 0} \frac{\partial P}{\partial z_{j}}(z) .
$$

Observe que, si $S \in \mathbb{C}\left[\left[z_{1}, \ldots, z_{n}\right]\right], \quad$ entonces $\quad \frac{\partial S}{\partial z_{j}} \in \mathbb{C}\left[\left[z_{1}, \ldots, z_{n}\right]\right] \quad$ y $\operatorname{ord}\left(\frac{\partial S}{\partial z_{j}}\right)=\operatorname{ord}(S)-1$

El gradiente $\nabla S$ de $S \in \mathbb{C}\left[\left[z_{1}, \ldots, z_{n}\right]\right]$ se define como: 


$$
\nabla S=\left(\frac{\partial S}{\partial z_{1}}, \ldots, \frac{\partial S}{\partial z_{n}}\right)
$$

No es difícil probar que con estas definiciones, el cálculo de polinomios puede generalizarse de manera natural a las series de potencias formales. Por ejemplo,

$$
\begin{aligned}
\frac{\partial(S \pm T)}{\partial z_{j}} & =\frac{\partial S}{\partial z_{j}} \pm \frac{\partial T}{\partial z_{j}} \\
\frac{\partial(c S)}{\partial z_{j}} & =c \frac{\partial S}{\partial z_{j}} \\
\frac{\partial(S \cdot T)}{\partial z_{j}} & =\frac{\partial S}{\partial z_{j}} \cdot T+S \cdot \frac{\partial T}{\partial z_{j}} \\
\nabla(S \pm T) & =\nabla S \pm \nabla T \\
\nabla(c S) & =c \nabla S \\
\nabla(S \cdot T) & =T \cdot \nabla S+S \cdot \nabla T,
\end{aligned}
$$

donde, $T \cdot \nabla S=\left(T \cdot \frac{\partial S}{\partial z_{1}}, \ldots, T \cdot \frac{\partial S}{\partial z_{n}}\right)$.

\section{CAMPOS VECTORIALES FORMALES}

Un campo vectorial formal $Z$ es una $n$-upla

$$
Z=\left(Z_{1}, \ldots, Z_{n}\right)
$$

donde cada $Z_{j} \in \mathbb{C}\left[\left[z_{1}, \ldots, z_{n}\right]\right]$. Denotaremos por $\mathcal{X}=\mathcal{X}\left[\left[z_{1}, \ldots, z_{n}\right]\right]$ al conjunto de todos los campos vectoriales formales. Observe que,

$$
\mathcal{X}=\mathbb{C}\left[\left[z_{1}, \ldots, z_{n}\right]\right] \times \ldots \times \mathbb{C}\left[\left[z_{1}, \ldots, z_{n}\right]\right](n \text { veces })
$$

y si $S \in \mathbb{C}\left[\left[z_{1}, \ldots, z_{n}\right]\right]$, entonces $\nabla S \in \mathcal{X}$.

Sean $Z, W \in \mathcal{X}$, con $Z=\left(Z_{1}, \ldots, Z_{n}\right)$ y $W=\left(W_{1}, \ldots, W_{n}\right), S=\sum_{|\alpha| \geq 0} a_{\alpha} z^{\alpha} \in \mathbb{C}\left[\left[z_{1}, \ldots, z_{n}\right]\right]$ y $c \in \mathbb{C}$. La suma $Z+W$, el producto por escalares $c Z$, el producto $S \cdot Z$, la composición de un campo con una serie $S \circ Z$ y la composición de campos $S \circ W$ se definen respectivamente como,

$$
\begin{aligned}
Z+W & =\left(Z_{1}+W_{1}, \ldots, Z_{n}+W_{n}\right) \\
c Z & =\left(c Z_{1}, \ldots, c Z_{n}\right) \\
S \circ Z & =\left(S \cdot Z_{1}, \ldots, S \cdot Z_{n}\right) \\
S \circ Z & =\sum_{|\alpha| \geq 0} a_{\alpha} Z^{\alpha} \\
Z \circ W & =\left(Z_{1} \circ W, \ldots, Z_{n} \circ W\right) .
\end{aligned}
$$


Si $Z=\left(Z_{1}, \ldots, Z_{n}\right) \in \mathcal{X}$, entonces definimos el orden de $Z$ como

$$
\operatorname{ord}(Z)=\min \left\{\operatorname{ord}\left(Z_{1}\right), \ldots, \operatorname{ord}\left(Z_{n}\right)\right\} .
$$

Por otro lado, como $Z_{j} \in \mathbb{C}\left[\left[z_{1}, \ldots, z_{n}\right]\right]$ entonces podemos escribir

$$
Z_{j}=\sum_{k \geq 0} Z_{j}^{k}
$$

donde cada $Z_{j}^{k}$ es un polinonio homogéneo de grado $k$. Si denotamos

$$
Z^{k}=\left(Z_{1}^{k}, \ldots, Z_{n}^{k}\right)
$$

entonces, $Z^{k}$ es un campo vectorial cuyas coordenadas son polinomios homogéneos de grado $k$ y se cumple

$$
Z=\sum_{k \geq 0} Z^{k}
$$

Observe que $\operatorname{ord}(Z)=r$ si y sólo si $Z^{0} \equiv Z^{1} \equiv \ldots \equiv Z^{r-1} \equiv 0$ y $Z^{r} \not \neq 0$.

El campo homogéneo $Z^{1}(z)=\left(Z_{n}^{1}(z), \ldots, Z_{n}^{1}(z)\right)$ es denominado parte lineal de $Z$ y puede ser representado por una matriz $A \in \mathbb{C}^{n \times n}$, es decir, $Z^{1}(z)=A z$.

Del mismo modo que en las series formales, las reglas del cálculo pueden ser extendidas de manera natural a los campos vectoriales formales. En efecto, la derivada de $Z=\left(Z_{1}, \ldots, Z_{n}\right)$ se define como

$$
Z^{\prime}=\frac{\partial\left(Z_{1}, \ldots, Z_{n}\right)}{\partial\left(z_{1}, \ldots, z_{n}\right)}=\left(\begin{array}{ccc}
\frac{\partial Z_{1}}{\partial z_{1}} & \cdots & \frac{\partial Z_{1}}{\partial z_{n}} \\
\vdots & \ddots & \vdots \\
\frac{\partial Z_{n}}{\partial z_{1}} & \cdots & \frac{\partial Z_{n}}{\partial z_{n}}
\end{array}\right)
$$

Es claro que, $Z^{\prime}(0) \in \mathbb{C}^{n \times n}$ es la matriz que representa a la parte lineal $Z^{1}$, es decir

$$
Z^{1}(z)=Z^{\prime}(0) z
$$

En lo que sigue, denotaremos por $\mathcal{P}^{n}$ (respectivamente $\mathcal{H}_{r}^{n}$ ) al conjunto de todos los campos formales $Z=\left(Z_{1}, \ldots, Z_{n}\right)$, tales que, $Z_{j} \in \mathcal{P}$ (respectivamente $Z_{j} \in \mathcal{H}_{r}$ ), es decir

$$
\mathcal{P}^{n}=\mathcal{P} \times \ldots \times \mathcal{P}(n \text { veces }) \text { y } \mathcal{H}_{r}^{n}=\mathcal{H}_{r} \times \ldots \times \mathcal{H}_{r}(n \text { veces }) .
$$

\section{Proposición 3.1.}

Si $P \in \mathcal{H}_{k}$ y $Q \in \mathcal{H}_{r}^{n}$, con $k, r>1$ entonces, 


$$
P \circ(I+Q)=P+R
$$

donde $R \in \mathcal{P}$ es tal que, ord $(R)>\max \{k, r\}$.

Demostración. Sea $P(z)=\sum_{|\alpha|=k} a_{\alpha} z^{\alpha} \in \mathcal{H}_{k} \quad$ y $\quad Q=\left(Q_{1}, \ldots, Q_{n}\right) \in \mathcal{H}_{r}^{n}$. Se tiene que

$$
\begin{aligned}
{[P \circ(I+Q)](z) } & =P(z+Q(z))=\sum_{|\alpha|=k} a_{\alpha}(z+Q(z))^{\alpha} \\
& =\sum_{|\alpha|=k} a_{\alpha}\left(\prod_{j=1}^{n}\left(z_{j}+Q_{j}(z)\right)^{\alpha_{j}}\right)
\end{aligned}
$$

Pero, dado $\alpha=\left(\alpha_{1}, \ldots, \alpha_{n}\right)$ multi-índice con $|\alpha|=k$ tenemos

$$
\left(z_{j}+Q_{j}(z)\right)^{\alpha_{j}}=z_{j}^{\alpha_{j}}+H_{j}(z),
$$

donde, $H_{j}(z)=C_{1} z_{j}^{\alpha_{j}-1} Q_{j}(z)+C_{2} z_{j}^{\alpha_{j}-2} Q_{j}(z)^{2}+\ldots+Q_{j}(z)^{\alpha_{j}} \in \mathcal{P}$ es tal que, $\operatorname{ord}\left(H_{j}\right)=r+\alpha_{j}-1$. Luego,

$$
\prod_{j=1}^{n}\left(z_{j}+Q_{j}(z)\right)^{\alpha_{j}}=\prod_{j=1}^{n}\left(z_{j}^{\alpha_{j}}+H_{j}(z)\right)=z^{\alpha}+H_{\alpha}(z),
$$

donde

$$
H_{\alpha}=\sum_{j=1}^{n} \frac{z^{\alpha}}{z_{j}^{\alpha j}} H_{j}(z)+\ldots+\prod_{j=1}^{n} H_{j}(z)
$$

es un polinomio tal que $\operatorname{ord}\left(H_{\alpha}\right) \geq k+r-1>\max \{k, r\}$. Reemplazando en (5) tenemos

$$
\begin{aligned}
{[P \circ(I+Q)](z) } & =\sum_{|\alpha|=k} a_{\alpha}\left(z^{\alpha}+H_{\alpha}(z)\right) \\
& =\sum_{|\alpha|=k} a_{\alpha} z^{\alpha}+\sum_{|\alpha|=k} a_{\alpha} H_{\alpha}(z) \\
& =P(z)+R(z)
\end{aligned}
$$

donde $R(z)=\sum_{|\alpha|=k} a_{\alpha} H_{\alpha}(z)$ es un polinomio de orden mayor que $\min \{k, r\}$.

\section{Corolario.}

Si $Z \in \mathcal{H}_{k}^{n}$ y $Q \in \mathcal{H}_{r}^{n}$, con $k, r>1$, entonces,

$$
Z \circ(I+Q)=Z+W
$$

donde $W \in \mathcal{P}^{n}$ es tal que $\operatorname{ord}(W)>\max \{k, r\}$. 
Demostración. Denotando $Z=\left(Z_{1}, \ldots, Z_{n}\right) \in \mathcal{H}_{k}^{n}$, entonces,

$$
Z \circ(I+Q)=\left(Z_{1} \circ(I+Q), \ldots, Z_{n} \circ(I+Q)\right) \text {. }
$$

Aplicando la Proposición anterior a cada componente $Z_{j} \circ(I+Q)$, el resultado se sigue.

\section{Proposición 3.2.}

Si $Z=\sum_{k \geq r} Z^{k}$ es un campo vectorial formal de orden $r \quad y \quad Q \in \mathcal{H}_{r}^{n}$ entonces

$$
Z \circ(I+Q)=Z^{r}+W
$$

donde $W \in \mathcal{X}$ es tal que ord $(W)>r$.

Demostración. Aplicando el Corolario anterior

$$
\begin{aligned}
Z \circ(I+Q) & =\sum_{k \geq r} Z^{k} \circ(I+Q)=\sum_{k \geq r}\left(Z^{k}+W_{k}\right) \\
& =Z^{r}+\sum_{k \geq r+1} Z^{k}+\sum_{k \geq r} W_{k} \\
& =Z^{r}+W
\end{aligned}
$$

donde $W=\sum_{k \geq r+1} Z^{k}+\sum_{k \geq r} W_{k} \in \mathcal{X}$, tal que $\operatorname{ord}(W)>r$.

\section{ECUACIONES DIFERENCIALES FORMALES}

A cada campo vectorial formal $Z=\left(Z_{1}, \ldots, Z_{n}\right)$ le podemos asociar un sistema de ecuaciones diferenciales ordinarias

$$
\mid \begin{array}{cc}
z_{1}^{\prime}= & Z_{1}\left(z_{1}, \ldots, z_{n}\right) \\
\vdots & \vdots \\
z_{n}^{\prime}= & Z_{n}\left(z_{1}, \ldots, z_{n}\right)
\end{array}
$$

el cual, usando la notación matricial, puede ser escrito como

$$
z^{\prime}=Z(z)
$$

en donde $z^{\prime}=\frac{d z}{d T}$ con $T \in \mathbb{C}$. En lo sucesivo, vamos a suponer que $\operatorname{ord}(Z)=1$.

\section{Proposición 4.1.}

Si $Q_{r} \in \mathcal{H}_{r}^{n}(r>1)$ entonces el cambio de coordenadas

$$
z=w+Q_{r}(w)
$$


transforma la Ecuación Diferencial

$$
z^{\prime}=A z+\sum_{k \geq r} Z^{k}(z)
$$

$e n$

$$
w^{\prime}=A w+\left[Z^{r}(w)-\left(Q_{r}^{\prime}(w) A w-A Q_{r}(w)\right)\right]+\tilde{W}(w)
$$

donde $\tilde{W} \in \mathcal{X}$. es tal que ord $(\tilde{W}) \geq r+1$.

Demostración. Derivando (8) con respecto a $T$, reemplazando en (9) y usando la Proposición 3.2 tenemos

$$
\begin{aligned}
\left(I+Q_{r}^{\prime}(w)\right) w^{\prime} & =A w+A Q_{r}(w)+\sum_{k \geq r} Z^{k}\left(w+Q_{r}(w)\right) \\
& =A w+A Q_{r}(w)+\sum_{k \geq r}\left[Z^{k} \circ\left(I+Q_{r}\right)\right](w) \\
& =A w+A Q_{r}(w)+Z^{r}(w)^{+}+W(w)
\end{aligned}
$$

en donde $W \in \mathcal{X}$ es tal que $\operatorname{ord}(w)>r$.

Multiplicando a ambos lados de la igualdad anterior por la matriz

$$
\left(I+Q_{r}^{\prime}(w)\right)^{-1}=I-Q_{r}^{\prime}(w)+Q_{r}^{\prime}(w)^{2}-\ldots
$$

tenemos

$$
\begin{aligned}
w^{\prime}= & A w+A Q_{r}(w)+Z^{r}(w)+W(w)-Q_{r}^{\prime}(w) A w- \\
& -Q_{r}^{\prime}(w) A Q_{r}(w)-Q_{r}^{\prime}(w) Z^{r}(w)-Q_{r}^{\prime}(w) W(w)+\ldots \\
= & A w+\left[Z^{r}(w)-\left(Q_{r}^{\prime}(w) A w-A Q_{r}(w)\right)\right]+\tilde{W}(w) .
\end{aligned}
$$

Donde $\tilde{W}(w)=W(w)-Q_{r}^{\prime}(w) A Q_{r}(w)-Q_{r}^{\prime}(w) Z^{r}(w)-Q_{r}^{\prime}(w) W(w)+\ldots \quad$ es un campo formal tal que $\operatorname{ord}(\tilde{W}) \geq r+1$.

\section{Corolario.}

Si $Q_{r} \in \mathcal{H}_{r}^{n}(r>1)$ entonces el cambio de coordenadas

$$
z=w+Q_{r}(w)
$$

transforma la Ecuación Diferencial

$$
z^{\prime}=A z+Z^{2}(z)+\ldots+Z^{r-1}(z)+Z^{r}(z)+\sum_{k \geq r+1} Z^{k}(z)
$$

donde $\tilde{W} \in \mathcal{X}$, es tal que, $\operatorname{ord}(\tilde{W}) \geq r+1$. 
Demostración. Basta observar que $Z^{k} \circ\left(I+Q_{r}\right)=Z^{k}+H$ donde $\operatorname{ord}(H)>r$.

Un cambio de coordenadas del tipo $z=w+H(w)$ es denominado perturbación de la identidad.

La Proposición 4.1 y su Corolario nos dicen que si $H$ es un campo polinomial homogéneo de grado $r$, el cambio de coordenadas (8) transforma el campo polinomial $Z^{r}(z)$ en $Z^{r}(w)-\left(Q_{r}^{\prime}(w) A w-A Q_{r}(w)\right)$ y deja invariante a $Z^{2}, \ldots, Z^{r-1}$. De esta manera, si queremos que la ecuación transformada no posea términos de grado $r$, debemos elegir convenientemente el polinomial $Q_{r}$. Esto motiva la siguiente definición.

\section{Definición 4.1.}

Dado $A \in \mathbb{C}^{n \times n}$ y $\quad r>1$, definimos el operador

$$
\begin{aligned}
L_{A}: \mathcal{H}_{r}^{n} & \rightarrow \mathcal{H}_{r}^{n} \\
Q & \mapsto L_{A}(Q),
\end{aligned}
$$

donde $L_{A}(Q)(z)=Q^{\prime}(z) A z-A Q(z)$.

Como $\mathcal{H}_{r}$ es un $\mathbb{C}$-espacio vectorial generado por,

$$
\left\{z^{\alpha}:|\alpha|=r\right\}
$$

se sigue que $\mathcal{H}_{r}^{n}$ es un $\mathbb{C}$-espacio vectorial cuya base es dada por

$$
\left\{z^{\alpha} e_{s}:|\alpha|=r, s=1, \ldots, n\right\}
$$

Donde, $e_{1}=(1,0, \ldots, 0), e_{2}=(0,1,0, \ldots, 0), \ldots, e_{n}=(0, \ldots, 0,1)$. Observe que $L_{A}$ es una transformación lineal de $\mathcal{H}_{r}^{n}$ en $\mathcal{H}_{r}^{n}$.

\section{Proposición 4.2.}

Si $A \in \mathbb{C}^{n \times n}$ es una matriz diagonal entonces la matriz que representa al operador lineal $L_{A}: \mathcal{H}_{r}^{n} \rightarrow \mathcal{H}_{r}^{n}$ también es diagonal. Más aún, si denotamos

$$
Q_{\alpha, s}(z)=z^{\alpha} e_{s} ; \quad|\alpha|=r, s=1, \ldots, n
$$

entonces,

$$
L_{A}\left(Q_{\alpha, s}\right)=\left[\langle\alpha, \lambda\rangle-\lambda_{s}\right] Q_{\alpha, s} .
$$

Donde $\alpha_{1}, \ldots, \alpha_{n}$ son los autovalores de la matriz A $y\langle\alpha, \lambda\rangle=\sum_{j=1}^{n} \alpha_{j} \lambda_{j}$. 
Demostración. Como $A \in \mathbb{C}^{n \times n}$ es una matriz diagonal con autovalores $\lambda_{1}, \ldots, \lambda_{n}$ concluimos que

$$
A=\left[\begin{array}{cccc}
\lambda_{1} & 0 & \cdots & 0 \\
0 & \lambda_{2} & \cdots & 0 \\
\vdots & \vdots & \ddots & \vdots \\
0 & 0 & \cdots & \lambda_{n}
\end{array}\right]
$$

Por definición, dados $|\alpha|=r$ y $s=1, \ldots, n$ tenemos

$$
L_{A}\left(Q_{\alpha, s}\right)(z)=Q_{\alpha, s}^{\prime}(z) A z-A Q_{\alpha, s}(z)
$$

Pero,

$$
\begin{aligned}
Q_{\alpha, s}^{\prime}(z) A z & =\left[\begin{array}{cccc}
0 & 0 & \cdots & 0 \\
\vdots & \vdots & & \vdots \\
\alpha_{1} \frac{z^{\alpha}}{z_{1}} & \alpha_{2} \frac{z^{\alpha}}{z_{2}} & \cdots & \alpha_{n} \frac{z^{\alpha}}{z_{n}} \\
\vdots & \vdots & & \vdots \\
0 & 0 & \cdots & 0
\end{array}\right] \cdot\left[\begin{array}{c}
\lambda_{1} z_{1} \\
\lambda_{2} z_{2} \\
\vdots \\
\lambda_{n} z_{n}
\end{array}\right] \\
& =\left[\begin{array}{c}
\left(\alpha_{1} \lambda_{1}+\cdots+\alpha_{n} \lambda_{n}\right) z^{\alpha} \\
\vdots \\
0
\end{array}\right]=\langle\alpha, \lambda\rangle z^{\alpha} e_{s}
\end{aligned}
$$

y

$$
\begin{aligned}
A Q_{\alpha, s}(z) & =\left[\begin{array}{cccc}
\lambda_{1} & 0 & \cdots & 0 \\
0 & \lambda_{2} & \cdots & 0 \\
\vdots & \vdots & \ddots & \vdots \\
0 & 0 & \cdots & \lambda_{n}
\end{array}\right] \cdot\left[\begin{array}{c}
0 \\
\vdots \\
z^{\alpha} \\
\vdots \\
0
\end{array}\right] \\
& =\left[\begin{array}{c}
0 \\
\vdots \\
\lambda_{s} z^{\alpha} \\
\vdots \\
0
\end{array}\right]=\lambda_{s} z^{\alpha} e_{s} .
\end{aligned}
$$

Reemplazando (15) y (16) en (14) tenemos

$$
L_{A}\left(Q_{\alpha, s}\right)(z)=\left[\langle\alpha, \lambda\rangle-\lambda_{s}\right] Q_{\alpha, s}(z)
$$


Como $\left\{Q_{\alpha s}:|\alpha|=r, s=1, \ldots, n\right\}$ es una base de $\mathcal{H}_{r}^{n}$, se sigue que la matriz que representa al operador $L_{A}$ es diagonal.

Sabemos que una matriz es inversible si y sólo si el cero no es autovalor de ella. Esta observación y la Proposición 4.2 conducen a la siguiente definición.

\section{Definición 4.2.}

Decimos que los autovalores $\lambda_{1}, \ldots, \lambda_{n}$ de la matriz $A \in \mathbb{C}^{n \times n}$ son resonantes si y sólo si existe $\alpha \in \mathbb{N}^{n}$ multi-índice con $|\alpha| \geq 2$ y existe $s \in\{1, \ldots, n\}$ tales que,

$$
\lambda_{s}=\langle\alpha, \lambda\rangle
$$

El número $|\alpha|$ es denominado orden de resonancia.

\section{Corolario 1.}

Si $A \in \mathbb{C}^{n \times n}$ es una matriz diagonal cuyos autovalores son no resonantes entonces el operador lineal $L_{A}: \mathcal{H}_{r}^{n} \rightarrow \mathcal{H}_{r}^{n}$ es inversible, para todo $r>1$.

Demostración. Se sigue inmediatamente de la Proposición 4.2 y la definición anterior.

\section{Corolario 2.}

Si $A \in \mathbb{C}^{n \times n}$ es una matriz diagonalizable y sus autovalores no presentan resonancias de orden $r \geq 2$, entonces dado $P \in \mathcal{H}_{r}^{n}$ existe una única solución $Q \in \mathcal{H}_{r}^{n}$ de la ecuación

$$
L_{A}(Q)=P \text {. }
$$

Demostración. Es Inmediata.

\section{RESULTADOS PRINCIPALES}

A continuación, probamos el Teorema de linealización de Poincaré el cual proporciona condiciones necesarias para que un campo vectorial formal pueda ser linealizado por un cambio de coordenadas formal del tipo perturbación de la identidad.

\section{Teorema 5.1 (Teorema de Poincaré).}

Si $A \in \mathbb{C}^{n \times n}$ es una matriz diagonalizable y sus autovalores no presentan resonancias de orden $r \geq 2$, entonces la Ecuación Diferencial Formal

$$
z^{\prime}=A z+\sum_{k \geq 2} Z^{k}(z)
$$




$$
z=w+H(w)
$$

Donde $H \in \mathbb{C}\left[\left[w_{1}, \ldots, w_{n}\right]\right]$ con ord $(H) \geq 2$ en la Ecuación Lineal

$$
w^{\prime}=A w
$$

Demostración. Construiremos inductivamente la serie formal $H$.

Por hipótesis, los autovales de $A$ son no resonantes entonces en particular no presentan resonancias de orden 2. Como $Z^{2} \in \mathcal{H}_{2}^{n}$, por el Corolario 2 y la Proposición 4.2 existe un único $Q_{2} \in \mathcal{H}_{2}^{n}$ tal que $L_{A}\left(Q_{2}\right)=Z^{2}$. Por la Proposición 4.1, el cambio de coordenadas

$$
z=T_{1}\left(z^{1}\right)=z^{1}+Q_{2}\left(z^{1}\right)
$$

transforma la Ecuación Diferencial (17) en

$$
\left(z^{1}\right)^{\prime}=A z^{1}+Z_{(1)}^{3}\left(z^{1}\right)+z_{(1)}^{4}\left(z^{1}\right)+\ldots
$$

donde $Z_{(1)}^{k} \in \mathcal{H}_{k}^{n}, \forall k \geq 3$.

Nuevamente, por hipótesis los autovales de $A$ no presentan resonancias de orden 3. Como $Z_{(1)}^{3} \in \mathcal{H}_{2}^{n}$, por el Corolario 2 y la Proposición 4.2 existe un único $Q_{3} \in \mathcal{H}_{3}^{n}$ que $L_{A}\left(Q_{3}\right)=Z_{(1)}^{3}$. Por la Proposición 4.1, el cambio de coordenadas

$$
z^{1}=T_{2}\left(z^{2}\right)=z^{2}+Q_{3}\left(z^{2}\right)
$$

transforma la Ecuación Diferencial (21) en

$$
\left(z^{2}\right)^{\prime}=A z^{2}+Z_{(2)}^{4}\left(z^{2}\right)+z_{(2)}^{5}\left(z^{2}\right)+\ldots,
$$

donde $Z_{(2)}^{k} \in \mathcal{H}_{k}^{n}, \forall k \geq 4$. Siguiendo el proceso inductivamente concluimos que el cambio formal de coordenadas

$$
\begin{aligned}
w & =T(z)=\lim _{k \rightarrow \infty}\left(T_{k} \circ T_{k-1} \circ \ldots \circ T_{1}\right)(z) \\
& =\lim _{k \rightarrow \infty}\left(\left(I-Q_{k+1}\right) \circ\left(I-Q_{k}\right) \circ \ldots \circ\left(I-Q_{2}\right)\right)(z),
\end{aligned}
$$

transforma la Ecuación Diferencial (17) en la Ecuación Lineal (19). Finalmente, no es difícil probar que el cambio de coordenadas $T$ así construido es de la forma $I+H$ donde $H$ es una serie formal de orden $\geq 2$.

El Teorema de Poincaré establece que, si los autovalores de la parte lineal de un campo vectorial formal no presentan resonancias, entonces el campo puede ser linealizado por un cambio de coordenadas formal del tipo perturbación de la identidad. A continuación veremos que sucede cuando los autovalores presentan resonancias. 
Como hicimos en la sección 4, denotemos $Q_{\alpha, s}(z)=z^{\alpha} e_{s} \in \mathcal{H}_{r}^{n}$, donde $(|\alpha|=r \quad$ y $s=1, \ldots, n)$. Recordemos que

$$
\left\{Q_{\alpha, s}:|\alpha|=r, \quad s=1, \ldots, n\right\}
$$

es una base de $\mathcal{H}_{r}^{n}$. Luego, dado $P=\sum_{s=1}^{n} \sum_{|\alpha|=r} a_{\alpha, s} Q_{\alpha, s} \in \mathcal{H}_{r}^{n}$, sea $Q=\sum_{s=1}^{n} \sum_{\alpha \mid=r} q_{\alpha, s} Q_{\alpha, s} \in \mathcal{H}_{r}^{n}$ tal que $L_{A}(Q)=P$. Por la Proposición 4.2 tenemos

$$
\begin{aligned}
\sum_{s=1|\alpha|=r}^{n} \sum_{\alpha, s} a_{\alpha, s} & =L_{A}\left(\sum_{s=1 \mid}^{n} \sum_{\alpha \mid=r} q_{\alpha, s} Q_{\alpha, s}\right) \\
& =\sum_{s=1}^{n} \sum_{\alpha \mid=r} q_{\alpha, s} L_{A}\left(Q_{\alpha, s}\right) \\
& =\sum_{s=1}^{n} \sum_{\alpha \mid=r}\left[\langle\alpha, \lambda\rangle-\lambda_{s}\right] q_{\alpha, s} Q_{\alpha, s}
\end{aligned}
$$

concluimos que

$$
\left[\langle\alpha, \lambda\rangle-\lambda_{s}\right] q_{\alpha, s}=a_{\alpha, s}
$$

donde, los $a_{\alpha, s} \in \mathbb{C}$ son fijados y los $q_{\alpha, s}$ son constantes a determinar.

De esta manera, si los autovalores $\lambda_{1}, \ldots, \lambda_{n}$ de la matriz diagonal $A$ admiten una resonancia de orden $r \geq 2$ entonces no podemos despejar el correspondiente $q_{\alpha, s}$. Esto motiva la siguiente definición.

\section{Definición 5.1.}

El espacio resonante de orden $r \geq 2$, denotado por $\mathcal{R}_{r}^{n}$ es el subespacio de $\mathcal{H}_{r}^{n}$ generado por todo los $Q_{\alpha, s}$ tales que $\lambda_{s}=\langle\alpha, \lambda\rangle$, donde $|\alpha|=r$.

Observe que si los autovalores $\lambda_{1}, \ldots, \lambda_{n}$ de A no admiten resonancias de orden $r \geq 2$ entonces $\mathcal{R}_{r}^{n}=(0)$.

\section{Lema 5.1}

Si los autovalores $\lambda_{1}, \ldots, \lambda_{n}$ de la matriz diagonal $A$ admiten autovalores de orden $r \geq 2$, entonces existe un cambio de coordenadas del tipo

$$
z=w+Q_{r}(w),
$$

donde $Q_{r} \in \mathcal{H}_{r}^{n}$ que transforma la Ecuación Diferencial

$$
z^{\prime}=A z+\sum_{k \geq r} Z^{k}(z)
$$


en

$$
w^{\prime}=A w+R^{r}(w)+\tilde{W}(w)
$$

Donde $R^{r} \in \mathcal{R}_{r}^{n}$ y $\tilde{W} \in \mathcal{X}$ es tal que, $\operatorname{ord}(\tilde{W}) \geq r+1$.

Demostración. Sea $Z^{r}=\sum_{s=1 \mid}^{n} \sum_{\alpha \mid=r} a_{\alpha, s} Q_{\alpha, s} \in \mathcal{H}_{r}^{n}$. Denotemos

$$
S=\left\{(\alpha, s):|\alpha|=r, \quad s=1, \ldots, n \text { y } \lambda_{s}=\langle\alpha, \lambda\rangle\right\}
$$

y definimos

$$
Q_{r}=\sum_{(\alpha, s) \notin S} \frac{a_{\alpha, s}}{\langle\alpha, \lambda\rangle-\lambda_{s}} Q_{\alpha, s} \in \mathcal{H}_{r}^{n}
$$

Un cálculo sencillo prueba que,

$$
Z^{r}-L_{A}\left(Q_{r}\right)=\sum_{(\alpha, s) \in S} a_{\alpha, s} Q_{\alpha, s} \in \mathcal{R}_{r}^{n} .
$$

Denotado $R^{r}=Z^{r}-L_{A}\left(Q_{r}\right)$ y por la Proposición 4.1 el Lema se sigue.

\section{Teorema 5.2 (Teorema de Poincaré-Dulac).}

Si $A \in \mathbb{C}^{n \times n}$ es una matriz diagonalizable, entonces la Ecuación Diferencial Fomal

$$
z^{\prime}=A z+\sum_{k \geq 2} Z^{k}(z)
$$

es transformada, por un cambio formal de coordenadas del tipo

$$
z=w+H(w)
$$

(donde $H \in \mathbb{C}\left[\left[w_{1}, \ldots, w_{2}\right]\right]$ con $\operatorname{ord}(H) \geq 2$ ) en la ecuación

$$
w^{\prime}=A w+\sum_{k \geq 2} R^{k}(w)
$$

donde $R^{k} \in \mathcal{R}_{k}^{n}, \forall k \geq 2$.

Demostración. De manera análoga a la demostración del Teorema 5.1, construiremos $H$ por inducción. Como $Z^{2} \in \mathcal{H}_{2}^{n}$, existen dos alternativas:

1) Los autovalores de $A$ no admiten resonancias de orden 2. En este caso sabemos que existe un único $Q_{2} \in \mathcal{H}_{2}^{n}$ tal que $L_{A}\left(Q_{2}\right)=Z^{2}$ y por la Proposición 4.1, el cambio de coordenadas

$$
z=T_{1}\left(z^{1}\right)=z^{1}+Q_{2}\left(z^{1}\right)
$$

transforma la Ecuación Diferencial (28) en 


$$
\left(z^{1}\right)^{\prime}=A z^{1}+Z_{(1)}^{3}\left(z^{1}\right)+Z_{(1)}^{4}\left(z^{1}\right)+\ldots
$$

donde $Z_{(1)}^{k} \in \mathcal{H}_{k}^{n}, \forall k \geq 3$.

2) Los autovalores de $A$ admiten resonancias de orden 2. Por el Lema 5.1 existe un $Q_{2} \in \mathcal{H}_{2}^{n}$ tal que $L_{A}\left(Q_{2}\right)-Z^{2}=R^{2} \in \mathcal{R}_{2}^{n}$ y por la Proposición 4.1, el cambio de coordenadas

$$
z=T_{1}\left(z^{1}\right)=z^{1}+Q_{2}\left(z^{1}\right)
$$

transforma la Ecuación Diferencial (28) en

$$
\left(z^{1}\right)^{\prime}=A z^{1}+R^{2}\left(z^{1}\right)+Z_{(1)}^{3}\left(z^{1}\right)+Z_{(1)}^{4}\left(z^{1}\right)+\ldots
$$

donde $R^{r} \in \mathcal{R}_{r}^{n}$ y $Z_{(1)}^{k} \in \mathcal{H}_{r}^{n}, \forall k \geq 3$.

En cualquier caso (en presencia o ausencia de resonancias), existe un cambio de coordenadas $z=T_{1}\left(z^{1}\right)=z^{1}+Q_{2}\left(z^{1}\right)$ que transforma (28) en (34) (donde hacemos $R^{2}=0$ si no hay resonancia de orden 2). Prosiguiendo inductivamente y teniendo en cuenta que los cambios de coordenadas del tipo $I+Q_{r}$ no afectan los términos de grado menor que $r$ (ver Corolario de la Proposición 4.1), la prueba sigue.

\section{REFERENCIAS BIBLIOGRÁFICAS}

[1] Arnold V. I., Geometrical Methods in the Theory of Ordinary Differential Equations, Second Edition, Springer, GMW 250, (1988).

[2] Benazic R., Singularidades de Campos Vectoriales Holomorfos en el Dominio de Poincaré, Pro Mathematica, Vol. X, No 19-20, pp. 9-33, (1996),

[3] Benazic R., Estructura Local de los Puntos de Tangencia del Transformado Estricto de una Foliación por curvas en dimensión 3, PESQUIMAT, Vol. VI, pp. 1-20, (2003).

[4] Camacho C., Holomorphic Dinamical Systems. Summer School on Dynamical Systems (1st - 25th august 1983) Trieste P.O.B. 586 Italy.

[5] Camacho C., Lectures on Complex 2 - Dimensional Dynamical Systems. Summer School on Dynamical Systems (1st - 25th august 1983) Trieste SMR. 296/7 Italy.

[6] Camacho C. - P. Sad, Pontos Singulares de Equações Diferenciais Analíticas. $16^{\circ}$ Coloquio Brasileiro de Matemática, IMPA, (1987).

\section{Agradecimientos}

Los autores desean expresar su agradecimiento al Consejo Superior de Investigaciòn de la UNMSM y al CONCYTEC por el soporte económico y al Instituto de Matemática y Ciencias Afines -IMCA de la UNI por el excelente ambiente de trabajo. 